\title{
Growth of lipid vesicle structures: From surface fractals to mass fractals
}

\author{
Sándalo Roldán-Vargas, ${ }^{1}$ Ramon Barnadas-Rodríguez, ${ }^{2,3}$ Alberto Martín-Molina, ${ }^{1}$ Manuel Quesada-Pérez, ${ }^{4}$ \\ Joan Estelrich, ${ }^{2}$ and José Callejas-Fernández ${ }^{1, *}$ \\ ${ }^{1}$ Grupo de Física de Fluidos y Biocoloides, Departamento de Física Aplicada, Universidad de Granada, E-18071 Granada, Spain \\ ${ }^{2}$ Departament de Fisicoquímica, Facultat de Farmàcia, Universitat de Barcelona, E-08028 Barcelona, Catalonia, Spain \\ ${ }^{3}$ Centre d'Estudis en Biofísica, Facultat de Medicina, Universitat Autònoma de Barcelona, \\ E-08193 Cerdanyola del Vallès Bellaterra, Barcelona, Catalonia, Spain \\ ${ }^{4}$ Departamento de Física, Escuela Politécnica Superior de Linares, Universidad de Jaén, Linares, E-23700 Jaén, Spain
}

(Received 5 November 2007; published 23 July 2008)

\begin{abstract}
We study fractal vesicle aggregates whose morphology is conditioned by the interaction between the lipid vesicle membranes and calcium and magnesium ions. These morphologies are probed by means of static light scattering using a cross-correlation scheme that avoids the multiple intracluster scattering. In contrast to the branched structures induced by calcium, we report a singular surface- to mass-fractal transition controlled by the magnesium concentration. From infrared spectroscopy data we conclude that the specific dehydration of the lipid membranes due to these cations plays an essential role in short-range intervesicle interactions.
\end{abstract}

DOI: 10.1103/PhysRevE.78.010902

PACS number(s): 87.16.dr, 87.64.Cc, 82.70.-y

Lipid-divalent cation interactions have a great relevance in many biological processes. More specifically, the aggregation and fusion of lipid vesicles (liposomes) are intimately related to the capability of some ions to bind to phospholipid headgroups and form dehydrated intermembrane complexes [1-5]. Unfortunately, this scenario is not well understood yet since it depends intimately on the lipid-ion combination $[4,5]$. However, an analysis based on the fractal morphology of the clusters in aggregating liposome suspensions can enhance the comprehension of such interactions.

Fractal geometry offers helpful scaling concepts to characterize the cluster morphology of aggregating systems. In this formalism, many of the structures formed in colloid systems have been identified as mass fractals. Their mass $M$ scales with their radius $R$ as $M \propto R^{d_{f}}\left(1 \leqslant d_{f}<3\right)$ [6] and the fractal dimension $\left(d_{f}\right)$ depends strongly on the short-range interparticle interactions. In particular, tenuous clusters $\left(1.75 \leqslant d_{f} \leqslant 2.1\right)$ have been reported in irreversible aggregation [7], whereas dense cluster morphologies (even $d_{f}>2.5$ ) have been observed when internal restructuring is allowed $[8,9]$. In contrast to mass fractals, a second class of scaling behavior has been described for uniform objects $\left(d_{f}=3\right)$ limited by a fractal rough surface $S$ that scales with the radius of the object as $S \propto R^{d_{s}}\left(2 \leqslant d_{s}<3\right)$ [10]. These objects are known as surface fractals, and $d_{s}$ is their surface fractal dimension. Although the first reports on them are far in time [11], these structures have been rarely documented since then, perhaps due to the plausible misinterpretations of some suspiciously high $d_{f}$ values found in previous aggregation experiments.

In this work, we investigate the connection between cluster morphology and short-range interparticle interactions in suspensions of aggregating liposomes in the presence of two archetypal cations, calcium and magnesium [4]. In addition, from static light scattering (SLS) measurements, we report the first observation (as far as we know) of a transition from

\footnotetext{
*jcalleja@ugr.es
}

surface fractal to mass fractal structures in a suspension of aggregating vesicles. This morphological evolution is controlled by the amount of magnesium added and presents a final stable value of $d_{f}$ appreciably larger than that corresponding to a diffusion-limited cluster aggregation (DLCA) regime, $d_{f}=1.75$, which was previously reported in the presence of calcium [12]. To avoid spurious values due to the effect of multiple intracluster scattering (MIS) [13], our measured fractal dimensions were determined by means of an innovative treatment based on an experimental crosscorrelation scheme.

Our findings suggest that lipid-ion interactions are quite different for these two divalent cations: A strong attraction that retains the vesicles tightly bound and prevents internal rearrangement resulting in branched structures (calcium), and a weak attraction that allows bond bending with the consequent cluster compactification (magnesium). Our study and the proposal of these two different mechanisms are completed with the help of Fourier-transform infrared spectroscopy (FTIR). This analysis suggests that the hydration grade of the lipid molecule in the presence of these two divalent cations plays an essential role in the vesicles short-range interaction and conditions the resulting cluster morphology.

Formally, if the aggregates of a suspension show a fractal nature on length scales smaller than its mean radius $R$ and larger than its primary particle radius $a$, the structure factor $S(q)$, determined through a SLS measurement, presents a "power law" dependence on the magnitude of the scattering vector $q[14,15]$,

$$
\begin{gathered}
S(q) \propto q^{-\alpha} \\
\alpha=d_{f} \quad \text { (mass fractals), } \quad \alpha=6-d_{s} \quad \text { (surface fractals) }
\end{gathered}
$$

$\left(R^{-1} \ll q \ll a^{-1}\right)$. In the present work, our primary particles are liposomes composed of pure bovine brain phosphatidylserine (PS). Their mean external radius, established from a hollow sphere model via SLS measurements, was $a=75 \mathrm{~nm}$ (relative 
standard deviation of 0.2). This size and a unilamellar thickness of $4.5 \mathrm{~nm}$, contrasted by means of small angle x-ray scattering, typify our liposomes as large unilamellar vesicles.

The light scattering experiments were performed with a three-dimensional light scattering spectrometer with two incident He-Ne laser beams $(\lambda=632.8 \mathrm{~nm})$, LS instruments (Fribourg, Switzerland). A digital correlator computes the cross-correlation function of the registered scattered intensities, detected by two avalanche photodiodes, for which the time-dependent contributions of multiple scattered photons can be neglected. According to this design, and omitting multiplicative factors, we adopted the determination of an "effective" structure factor $S^{*}(q)$ given by [16]

$$
S^{*}(q)=\sqrt{\frac{\left\langle I_{1}^{a}(q, t)\right\rangle\left\langle I_{2}^{a}(q, t)\right\rangle}{\left\langle I_{1}^{0}(q, t)\right\rangle\left\langle I_{2}^{0}(q, t)\right\rangle}} \frac{\beta^{a}(q)}{\beta^{0}(q)},
$$

where $\left\langle I_{i}^{a}(q, t)\right\rangle$ is the total time-averaged intensity registered by detector $i$ for the aggregated sample for a fixed $q$ value, whereas $\left\langle I_{i}^{0}(q, t)\right\rangle$ is the intensity corresponding to a dilute nonaggregated suspension. In the same way, $\beta^{\text {system }}(q)$ (system $\in\{a, 0\}$ ) is the ratio of single and total scattered intensities for a given system. Therefore, the function $\beta^{a}(q) / \beta^{0}(q)$ corrects the magnitude of the structure factor due to the disturbance of multiple scattering [16]. Its experimental access is

$$
\left[\frac{\beta^{a}(q)}{\beta^{0}(q)}\right]^{2}=\frac{g_{12}^{a}(q, \tau=0)-1}{g_{12}^{0}(q, \tau=0)-1},
$$

where $g_{12}^{\text {system }}(q, \tau)=\frac{\left\langle I_{Y}^{\text {system }}(q, t) I_{2}^{\text {system }}(q, t+\tau)\right\rangle}{\left\langle I_{Y}^{\text {system }}(q, t)\right\rangle\left\langle I_{2}^{\text {ystem }}(q, t)\right\rangle}$ is the normalized cross-correlation function of the registered intensities.

The aggregation processes were initiated by mixing the appropriate salt solution, $\mathrm{MgCl}_{2}$, and the liposomes (suspended in purified water) simultaneously into a cylindrical scattering cell. The stationarity of the first cumulant of the autocorrelation function of the intensity was verified via dynamic light scattering (DLS) for $\left[\mathrm{MgCl}_{2}\right]<4 \mathrm{mM}$. Beyond this threshold aggregating concentration, all the examined samples were prepared to have a final liposome volume fraction of $0.1 \%$, over the range $5-150 \mathrm{mM}$ of magnesium. To obtain a reliable determination of $S^{*}(q)$ for each electrolyte concentration, the aggregation processes were stopped at different stadiums until a constant value for the fitting scattering exponent $\alpha$ was reached [expressions (1) and (2)]. The stops were effective by diluting the evolving system below the threshold aggregating salt concentration. At their final states, the apparent mean hydrodynamic radius of the aggregates exceeded $1.5 \mu \mathrm{m}$ for all the samples. The reliability of the fitting $q$ range was tested by reproducing satisfactorily the DLCA fractal dimension $\left(d_{f}=1.75\right)$ obtained in our previous study at $5 \mathrm{mM}$ of calcium [12]. This diffusive regime also had been proved from aggregation kinetics data obtained via independent DLS measurements. The $\alpha$ exponents determined with this protocol at $25^{\circ} \mathrm{C}$ for the considered electrolyte-liposome samples are summarized in Fig. 1. A clear tendency is revealed: The scattering exponents decrease with increasing the magnesium concentration reaching a permanent value in the vicinity of $30 \mathrm{mM}$. However, these re-

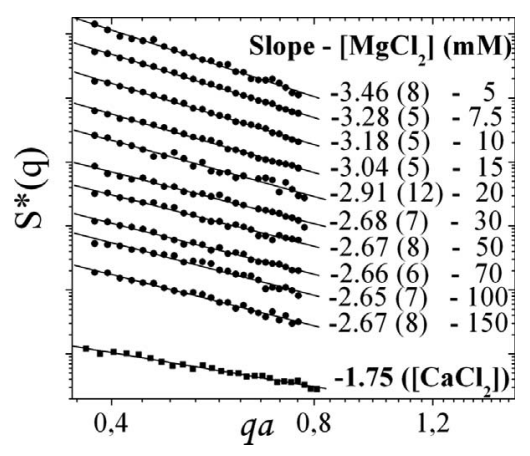

FIG. 1. Effective structure factors for the different magnesium concentrations. The experimental values (circles) result from expression (2), whereas solid lines represent the theoretical fit according to expression (1). The absolute error associated with each slope appears in parentheses and affects the last digits. Experimental structure factor at $5 \mathrm{mM}$ of $\mathrm{CaCl}_{2}$ (squares) [12]

sults deserve additional discussion concerning the a priori not negligible effects of the aggregate polydispersity and MIS on the measured $\alpha$ exponents.

Martin et al. [17] revealed (via DLS) that the regime of polydispersity of an aggregation process can introduce a disturbance on the $\alpha$ exponents. This disturbance can be contrasted through a strong $q$ dependence of the apparent mean translational diffusion coefficient $D_{t}^{a p p}(q)$ of the cluster distribution, for $q R>1$. Our evaluations showed that the largest relative deviation of $D_{t}^{a p p}(q)$ within the $q$-range $\left[6 \times 10^{-3}, 2.6 \times 10^{-2}\right] \mathrm{nm}^{-1} \quad$ was $\quad 30 \% \quad\left[\mid D_{t}^{a p p}\left(q_{\max }\right)\right.$ $-D_{t}^{a p p}\left(q_{\text {min }}\right) \mid / D_{t}^{a p p}\left(q_{\min }\right) \approx 0.3$ at the most]. This moderate $q$ dependence suggests a weak regime of polydispersity, for which an irrelevant effect on the scattering exponents is expected. In relation to the MIS (in a crude way) it will not affect the $\alpha$ exponents if the ratio $\beta^{a}(q) / \beta^{0}(q)$ is constant on $q$ [expression (2)]. The only suspension in which we found a clear $q$ dependence for $\beta^{a}(q) / \beta^{0}(q)$ was the $5 \mathrm{mM}$ sample (and very slightly at $7.5 \mathrm{mM}$ ). The rest of the samples exhibited an influence of MIS but as a common constant contribution $\left[\beta^{a}(q) / \beta^{0}(q) \approx\right.$ constant $\left.<1\right]$ over the explored $q$ range, not affecting significantly the values of the scattering exponents. Figure 2 shows these tendencies for the 5, 7.5, 10, 30 , and $100 \mathrm{mM}$ samples. At low salt concentrations, the

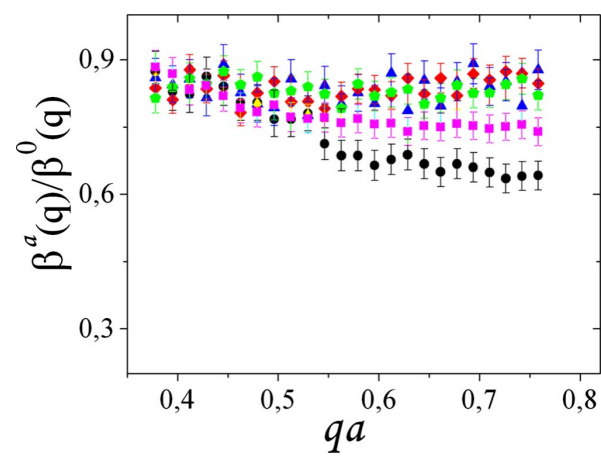

FIG. 2. (Color online) Experimental ratio $\beta^{a}(q) / \beta^{0}(q)$ from expression (3). $\left[\mathrm{MgCl}_{2}\right]$ : $5 \mathrm{mM}$ (black circles), $7.5 \mathrm{mM}$ (magenta squares), $10 \mathrm{mM}$ (blue triangles), $30 \mathrm{mM}$ (red diamonds), and $100 \mathrm{mM}$ (green pentagons). 
MIS, accounted for by $\beta^{a}(q) / \beta^{0}(q)$, presents a relative increment on $q$. If the MIS correction is not used, this effect takes us to the mistaken conclusion that we are in presence of rougher surfaces [e.g., $\alpha^{\text {spurious }}(5 \mathrm{mM})=3.12$ without MIS correction].

Accordingly, the power-law behaviors exhibited by $S^{*}(q)$ for all the samples (Fig. 1) would correspond to welldetermined fractal morphologies. Certainly, the local vesicle deformation due to bending elasticity forces [18] and the nondispensable appearance of fusion should be considered, however the power-law behaviors (even at $q \approx a^{-1}$ ) are consistent with a nondistortion of the form factor of the primary vesicles. Hence we interpret our results as a morphological evolution of the fractal aggregates with $\left[\mathrm{MgCl}_{2}\right]$. Starting at $5 \mathrm{mM}$ of magnesium, with $d_{s}=6-\alpha(5 \mathrm{mM})=2.54 \pm 0.08$, the resulting structures are included into the surface fractals domain. The increasing roughness takes us to the mass fractals domain $\left(\left[\mathrm{MgCL}_{2}\right]>15 \mathrm{mM}, \alpha<3\right)$ in which, finally, a stable (and dense) ramification, $d_{f}=2.6-2.7$, remains over a long range of electrolyte concentration $\left(30<\left[\mathrm{MgCl}_{2}\right]\right.$ $\leqslant 150 \mathrm{mM}$ ). Certainly, very few structures of biological interest have been described previously by the notion of surface fractal [19] since the existing works usually are concerned with the study of the porosity of rough particles. For instance, the pioneering results obtained by Keefer and Schaefer [11] in a system of rough polycondensates of silica show a clear analogy with our measured fractal dimensions, even though they studied a significantly different system.

The results in the presence of magnesium are also contrasted in Fig. 1 with those reported in our previous study in the presence of calcium, for which the lack of vesicle fusion had been verified by means of fluorescence essays [12]. A brief compendium of these morphological differences suggests a simplified interaction picture. In our previous study, two limiting irreversible regimes were documented in the aggregation induced by calcium [12]: A slow regime (2.5 $\left.\leqslant\left[\mathrm{CaCl}_{2}\right]<5 \mathrm{mM}\right)$, and a DLCA regime $\left(5 \leqslant\left[\mathrm{CaCl}_{2}\right]\right.$ $\leqslant 7 \mathrm{mM}$ ). In both regimes, the energy of the bond between two vesicles after sticking would be great enough to prevent cluster compactification. It is the increase of the probability of sticking with $\left[\mathrm{CaCl}_{2}\right]$, attributed to the reduction of a residual repulsive barrier at short distances, which established in that case a range from 1.91 to 1.75 for the fractal dimension of the resulting structures. On the contrary, aggregation induced by magnesium produces at any concentration very dense clusters that cannot be explained without contemplating restructuring effects and, consequently, a weak bond between two bound vesicles. In this situation, the origin of the morphological evolution between 5 and $30 \mathrm{mM}$ can be understood by the strengthening of this bond with $\left[\mathrm{MgCl}_{2}\right]$. Indeed, in the case of a weak bond, a vesicle will be fixed on the surface of a structure if the number of bonds with its nearest neighbors is high. As the bond becomes stronger, the number of them necessary to fix a vesicle will decrease, increasing the ramification of the structure. This process is detained when the cluster ramification remains stable.

This scenario cannot be discussed by appealing to a stabilization model based only on a Coulombic repulsive interaction. Accordingly, several authors have pointed out the dehydration effect of the lipid headgroups exerted by metal

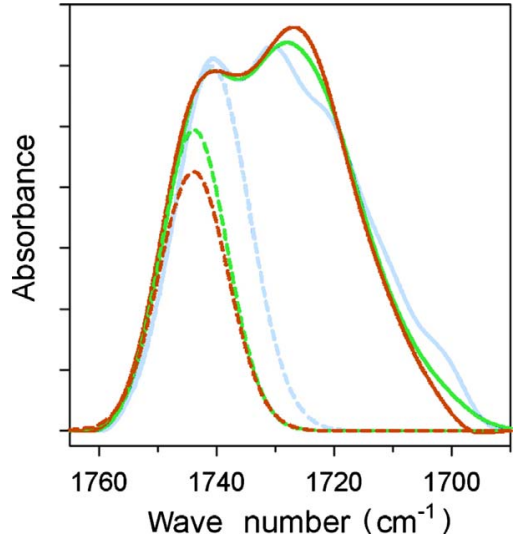

FIG. 3. (Color online) FTIR spectra of carbonyl band of PS liposomes. Continuous lines: Deconvolved spectra in presence of 0 (dark red), 2.5 (medium green), and $5 \mathrm{mM}$ (light cyan) of $\mathrm{CaCl}_{2}$. Discontinuous lines: Dehydrated component bands of the previous spectra. The total areas are normalized.

cations as a fundamental mechanism in aggregation and fusion processes in vesicle suspensions [20-22]. In this respect, we used FTIR spectroscopy in order to investigate this effect. Thus, we acquired FTIR spectra of our liposomes prepared in $\mathrm{D}_{2} \mathrm{O}$, containing the appropriate amount of the desired cations, with a spectrometer (Mattson Polaris). Each spectrum was obtained at a resolution of $2 \mathrm{~cm}^{-1}$ using two $\mathrm{CaF}_{2}$ windows with a spacer containing the sample. The final intensity of the infrared absorption was quantified subtracting the spectra of the corresponding solvent acquired under the same conditions. We focused our attention on the stretching band of the carbonyl ester, which has proved to be very sensitive to the amount of water bounded to the phospholipid molecule, i.e., to the hydration grade [23]. This stretching band presents a fine structure composed by minor bands corresponding to the hydrated and nonhydrated carbonyl ester groups. We analyzed the contribution of the nonhydrated carbonyl band to this multicomponent band quantifying its relative area after Fourier deconvolution. Our results showed that, when $\mathrm{CaCl}_{2}$ was added to the aqueous medium, there was an increase of the area of the nonhydrated carbonyl band, which showed no significant shift of its maximum at any salt concentration assayed. At $2.5 \mathrm{mM}$ of $\mathrm{CaCl}_{2}$ this band had an area $16 \%$ larger than that in pure $\mathrm{D}_{2} \mathrm{O}$. A plateau was reached at $5 \mathrm{mM}$ of $\mathrm{CaCl}_{2}$, being the area, in this case, $62 \%$ higher than that in $\mathrm{D}_{2} \mathrm{O}$ (discontinuous lines in Fig. 3). Consequently, no further change occurs between 5 and $7 \mathrm{mM}$ of $\mathrm{CaCl}_{2}$. On the other hand, equivalent essays were performed in the presence of $\mathrm{MgCl}_{2}$ over the range 0-20 mM. In that case, the studied carbonyl peak showed no significant change of the percentage area of its dehydrated carbonyl band within the studied range.

Therefore, as the calcium concentration increases, the progressive dehydration of the carbonyl group would be correlated with the reduction of the repulsive barrier at the slow regime mentioned before. Then, we might assume that this hydration grade which is coupled with the unscreened Coulombic repulsion (at least at low calcium concentrations), would act as a repulsive barrier. In the case of magnesium, 
the internal dehydration of the lipid membrane associated to the carbonyl group would not take place. Hence, this nonsuppressed hydration would reduce the depth of the energy minimum and would increase the distance between two bound vesicles, so cluster compactification is enhanced. However, the fractal evolution and the later stabilization of the fractal dimension in the presence of magnesium require further investigations. In our opinion, a more exhaustive study of the fine structure of the infrared absorption associated to the chemical groups of the phospholipid molecule in the presence of this cation can throw some light on these questions. In any case, our observations, supported on the analysis of the spectra of the lipid headgroups, may be consistent with a model based on the addition of Coulombic and hydration repulsions, together with van der Waals attraction, as that presented by Ohki and Arnold [4].

In conclusion, our morphological analysis of vesicle aggregates together with FTIR data suggest that the specific dehydration of the lipid membranes induced by different cations should be considered as an essential element in the short-range intervesicle interaction. As a result of the hydration repulsive interaction, a variety of fractal vesicle structures scarcely present in colloid systems emerges: surface fractals. From light scattering experiments performed by means of a cross-correlation technique, we have found a rarely reported surface- to mass-fractal transition of liposome aggregates, which is controlled by the magnesium concentration. This contrasts with the classical mass-fractal structures induced by calcium.

The authors acknowledge the MEC (Projects No. MAT2006-12918-C05-01, No. -02, and No. -05), ERDF Funds, and "Junta de Andalucía" (Projects No. P07-FQM2496 and No. P07-FQM-02517). A.M.-M. also acknowledges the "Programa Ramón y Cajal 2005, MEC-EFS (Contract No. RYC-2005-000829)."
[1] D. D. Lasic, Handbook of Biological Physics (Elsevier, London, 1995).

[2] J. Wilschut and D. Hoekstra, Chem. Phys. Lipids 40, 145 (1986).

[3] D. Papahadjopoulos et al., J. Bioenerg. Biomembr. 22, 157 (1990).

[4] S. Ohki and K. Arnold, Colloids Surf., B 18, 83 (2000).

[5] H. Binder and O. Zschörnig, Chem. Phys. Lipids 115, 39 (2002).

[6] B. B. Mandelbrot, Fractals, Form, Chance and Dimension (Freeman, San Francisco, 1977).

[7] D. A. Weitz, J. S. Huang, M. Y. Lin, and J. Sung, Phys. Rev. Lett. 54, 1416 (1985).

[8] S. J. Jung, R. Amal, and J. A. Raper, Powder Technol. 88, 51 (1996).

[9] P. J. Lu, J. C. Conrad, H. M. Wyss, A. B. Schofield, and D. A. Weitz, Phys. Rev. Lett. 96, 028306 (2006).

[10] D. Avnir, D. Farin, and P. Pfeifer, J. Colloid Interface Sci. 103, 112 (1985).

[11] K. D. Keefer and D. W. Schaefer, Phys. Rev. Lett. 56, 2376
(1986).

[12] S. Roldan-Vargas et al., Phys. Rev. E 75, 021912 (2007).

[13] C. M. Sorensen, Aerosol Sci. Technol. 35, 648 (2001).

[14] D. W. Schaefer, J. E. Martin, P. Wiltzius, and D. S. Cannell, Phys. Rev. Lett. 52, 2371 (1984).

[15] H. D. Bale and P. W. Schmidt, Phys. Rev. Lett. 53, 596 (1984).

[16] C. Urban and P. Schurtenberger, J. Colloid Interface Sci. 207, 150 (1998).

[17] J. E. Martin and F. Leyvraz, Phys. Rev. A 34, 2346 (1986); J. E. Martin, J. P. Wilcoxon, D. Schaefer, and J. Odinek, ibid. 41, 4379 (1990).

[18] D. N. Petsev, Langmuir 15, 1096 (1999).

[19] J. Schüler et al., Biophys. J. 77, 1117 (1999).

[20] W. Hübner and A. Blume, Chem. Phys. Lipids 96, 99 (1998).

[21] T. Fukuma, M. J. Higgins, and S. P. Jarvis, Phys. Rev. Lett. 98 , 106101 (2007).

[22] M. L. Berkowitz, D. L. Bostick, and S. Pandit, Chem. Rev. (Washington, D.C.) 106, 1527 (2006).

[23] V. Buzón, E. Padrós, and J. Cladera, Biochemistry 44, 13354 (2005). 